\title{
Approximation of Complex-Valued Functions by Fractal Functions
}

\section{Approximation des fonctions à valeur complexe par les fonctions fractales}

\section{N. Vijender}

Department of Mathematics

Visvesvaraya of National Institute of Technology Nagpur

Nagpur - 440010, India

vijendernallapu@gmail.com

\begin{abstract}
Fractal approximants developed through iterated function systems (IFS) prove more versatile than classical approximants. In this paper, we introduce a new class of fractal approximants using the suitable bounded linear operators defined on the space $\mathcal{C}(I)$ of continuous functions and concept of $\alpha$-fractal functions. The convergence of the proposed fractal approximants towards the original continuous function does not need any condition on the scaling factors. The fractal approximants proposed in this paper possess fractality and convergence simultaneously. Without imposing any condition on the scaling vector, we establish the constrained approximation by the proposed fractal approximants. Existence of Schauder basis of fractal polynomials for the space of continuous functions $\mathcal{C}(I)$ is investigated. Using the proposed class of fractal approximants, we develop complex fractal approximants for representation of the square integrable complexvalued functions defined on a real compact interval.

2010 Mathematics Subject Classification. 28A80, 28A10, 41A30, 41A17, $26 \mathrm{~A} 27$.

KEYWORDS. Fractal approximation, Complex fractal approximation, Fractal dimension, Constrained fractal approximation, Convergence.
\end{abstract}

\section{Introduction}

Barnsley [1] introduced the concept of fractal interpolation functions (FIFs) through iterated function system (IFS). Inspired by the Barnsley work, many researchers [2, 3] have developed various aspects of FIFs. The concept of FIF can be used to associate a family of functions to a given function $f$ defined on a real compact interval $I=\left[x_{1}, x_{N}\right]$ (for instance, see [1,4]). An element of this family is denoted by $f^{\alpha}$ and Navascués [4] named it as $\alpha$-fractal function associated with $f$. This function $f^{\alpha}$ contains a set of real parameters, namely, scaling factors. The fractality/irregularity associated with $f^{\alpha}$ can be measured through its fractal dimension [5]. Using the concept of $\alpha$-fractal functions various kinds of fractal approximants have been developed $[6,7,8,9,10,11,12,13,14]$ to obtain the fractal analogue of many classical approximation results. In this manuscript, we develop a new kind of fractal approximants that converge to the original function without losing fractality. Additionally, for a given continuous function $f$ defined on a real compact interval $I=\left[x_{1}, x_{N}\right]$, we develop a sequence of fractal functions that converges uniformly to $f$ even if the magnitude of the scaling factors does not go to zero.

For every $n \in \mathbb{N}$, let $b_{n}: \mathcal{C}(I) \rightarrow \mathcal{C}(I)$ be bounded and linear operator such that the following properties are satisfied for every $f \in \mathcal{C}(I)$.

$$
b_{n}(f)\left(x_{1}\right)=f\left(x_{1}\right), b_{n}(f)\left(x_{N}\right)=f\left(x_{N}\right) \text {, and }\left\|b_{n}(f)-f\right\|_{\infty} \rightarrow 0 \text { as } n \rightarrow \infty .
$$

There exist many operators which obey the above properties, for instance, Bernstein operator [15]. In this article, for a given function $f \in \mathcal{C}(I)$, by exploiting fractal approximation theory and taking $b_{n}(f), n \in$ $\mathbb{N}$, as base functions, we construct a sequence $\left\{f_{n}^{\alpha}\right\}_{n=1}^{\infty}$ of $\alpha$-fractal functions that converges uniformly 
to $f$ for every scaling vector. The convergence of the proposed fractal functions towards $f$ follows from the convergence of the sequence $\left\{b_{n}(f)\right\}_{n=1}^{\infty}$ towards $f$. The procedure of getting a sequence $\left\{f_{n}^{\alpha}\right\}_{n=1}^{\infty}$ of $\alpha$-fractal functions that converges uniformly to $f \in \mathcal{C}(I)$ determines a bounded and linear operator, termed $\alpha$-fractal operator: $\mathcal{F}_{n}^{\alpha}: \mathcal{C}(I) \rightarrow \mathcal{C}(I), f \rightarrow f_{n}^{\alpha}$.

Approximation of functions from below or above is called constrained approximation, and it is a well studied in the context of classical polynomial splines. By imposing the suitable conditions on the base function and scaling factors, the constrained approximation by the fractal functions is studied in $[16,17,18]$. In this paper, we study the constrained approximation by the proposed class of $\alpha$-fractal functions without imposing any condition on the scaling vector. In other words, constrained fractal approximants proposed in this paper work equally for all the scaling vectors. In particular, our constrained approximation results provide positivity preserving approximants.

For a given function $f \in \mathcal{C}(I)$, we develop non-smooth polynomials that approach $f$ without losing their fractality. We investigate the existence of Schauder basis of non-smooth polynomials for $\mathcal{C}(I)$. Constrained approximation by non-smooth polynomials is established. Using the $\alpha$-operator defined on $\mathcal{C}(I)$, we define $\alpha$-fractal operator on the space $\mathcal{C}(I, \mathbb{C})$ of complex-valued continuous functions defined on the real compact interval $I$. To construct complex fractal approximants, $\alpha$-fractal operator on the space $\mathcal{C}(I, \mathbb{C})$ is extended to a $\alpha$-fractal operator on the space of square integrable complex-valued functions defined on a compact real interval $I$.

\section{Background and preliminaries}

The following notation and terminologies will be used throughout the article. The set of real numbers will be denoted by $\mathbb{R}$, whilst the set of natural numbers by $\mathbb{N}$. For a fixed $N \in \mathbb{N}$, we shall write $\mathbb{N}_{N}$ for the set of first $N$ natural numbers. Given real numbers $x_{1}$ and $x_{N}$ with $x_{1}<x_{N}$, we define $\mathcal{C}\left[x_{1}, x_{N}\right]$ to be the space of all real-valued continuous functions on $I=\left[x_{1}, x_{N}\right]$.

Let $x_{1}<x_{2}<\cdots<x_{N-1}<x_{N}(N>2)$ be a partition of the closed interval $I=\left[x_{1}, x_{N}\right]$, and $y_{1}, y_{2}$, $\ldots, y_{N}$ be a collection of real numbers. Let $L_{i}, i=1,2, \ldots, N-1$, be a set of homeomorphism mappings from $I$ to $I_{i}=\left[x_{i}, x_{i+1}\right]$ satisfying $L_{i}\left(x_{1}\right)=x_{i}$, and $L_{i}\left(x_{N}\right)=x_{i+1}$. Let $F_{i}$ be a function from $I \times \mathbb{R}$ to $\mathbb{R}$, which is continuous in the $x$-direction and contractive in the $y$-direction such that $F_{i}\left(x_{1}, y_{1}\right)=y_{i}$, $F_{i}\left(x_{N}, y_{N}\right)=y_{i+1}, i \in \mathbb{N}_{N-1}$. Using the IFS $\mathcal{I}=\left\{I \times \mathbb{R}, w_{i}(x, y)=\left(L_{i}(x), F_{i}(x, y)\right), i \in \mathbb{N}_{N-1}\right\}$, Barnsley [1] proved that (i) there exists a continuous function $f^{*}: I \rightarrow \mathbb{R}$ satisfying $f^{*}\left(x_{i}\right)=y_{i}, i \in \mathbb{N}_{N}$, (ii) $f^{*}(x)=F_{i}\left(L_{i}^{-1}(x), f^{*} \circ L_{i}^{-1}(x)\right), x \in I_{i}$, and (iii) $G\left(f^{*}\right)=\underset{i \in \mathbb{N}_{N-1}}{\cup} w_{i}\left(G\left(f^{*}\right)\right)$, where $G\left(f^{*}\right)$ is the graph of $f^{*}$. The above function $f^{*}$ is fractal interpolation function (FIF) corresponding to the IFS $\mathcal{I}$.

Barnsley and Navascués [1, 6, 7] observed that the concept of FIFs can be used to define a class of fractal functions associated with a given real-valued continuous function $f$ on a compact interval $I$. For a given $f \in \mathcal{C}(I)$, consider a partition $\Delta=\left\{x_{1}, x_{2}, \ldots, x_{N}\right\}$ of $\left[x_{1}, x_{N}\right]$ satisfying $x_{1}<x_{2}<\cdots<$ $x_{N}$, a continuous function $b: I \rightarrow \mathbb{R}$ that fulfills the conditions $b\left(x_{1}\right)=f\left(x_{1}\right), b\left(x_{N}\right)=f\left(x_{N}\right)$ and $b \neq f$, and $N-1$ real numbers $\alpha_{i}, i \in \mathbb{N}_{N-1}$ satisfying $\left|\alpha_{i}\right|<1$. Define an IFS through the maps

$$
L_{i}(x)=a_{i} x+b_{i}, F_{i}(x, y)=\alpha_{i} y+f\left(L_{i}(x)\right)-\alpha_{i} b(x), i \in \mathbb{N}_{N-1} .
$$

The corresponding FIF denoted by $f_{\Delta, b}^{\alpha}=f^{\alpha}$ is referred to as $\alpha$-fractal function (fractal version of $f$ ) for $f$ with respect to a scaling vector $\alpha=\left(\alpha_{1}, \alpha_{2}, \ldots, \alpha_{N-1}\right)$, base function $b$, and partition $\Delta$. Here the 
set of data points is $\left\{\left(x_{i}, f\left(x_{i}\right)\right): i \in \mathbb{N}_{N}\right\}$. The $\alpha$-fractal function $f^{\alpha}$ corresponding to $f$ satisfies the self-referential equation

$$
f^{\alpha}(x)=\alpha_{i} f^{\alpha}\left(L_{i}^{-1}(x)\right)+f(x)-\alpha_{i} b\left(L_{i}^{-1}(x)\right), x \in I_{i}, i \in \mathbb{N}_{N-1} .
$$

The fractal dimension (box dimension or Hausdorff dimension) of $f^{\alpha}$ depends on the choice of the scaling vector $\alpha, f$, and base function $b$ (see for instance, Nasim Akhtar et. al [5]). According to Nasim Akhtar et. al [5]): if the data points $\left(x_{i}, f\left(x_{i}\right)\right), i \in \mathbb{N}_{N}$ are not collinear, then graph $G$ of the $\alpha$-fractal function $f^{\alpha}$ has the box dimension

$$
\operatorname{dim}_{B}^{G}=\left\{\begin{array}{l}
D \quad \text { if } \sum_{i=1}^{N-1}\left|\alpha_{i}\right|>1 \\
1 \quad \text { otherwise }
\end{array}\right.
$$

where $D$ is solution of $\sum_{i=1}^{N-1}\left|\alpha_{i}\right| a_{i}^{D-1}=1$.

The following remark explains about the sufficient condition for a $\alpha$-fractal function to be irregular (nondifferentiable) on $I$, and it is a consequence of Lemma 5.1 and Theorem 5.2 of [6].

Remark 2.1. Let $f \in \mathcal{C}^{1}(I)$. Let $\Delta=\left\{x_{1}, x_{2}, \ldots, x_{N}\right\}$ be a partition of $I=\left[x_{1}, x_{N}\right]$ satisfying $x_{1}<x_{2}<\cdots<x_{N}$ and $\alpha=\left(\alpha_{1}, \alpha_{2}, \ldots, \alpha_{N-1}\right)$. If $\sum_{i=1}^{N-1}\left|\alpha_{i}\right|>1$, then the set of points of nondifferentiability of $f^{\alpha}$ is dense in $I$.

From (2.2), the following bound for the uniform error in the process of $\alpha$-fractal approximation can be easily deduced:

$$
\left\|f^{\alpha}-f\right\|_{\infty} \leq \frac{|\alpha|_{\infty}}{1-|\alpha|_{\infty}}\|f-b\|_{\infty}, \text { where }|\alpha|_{\infty}=\max \left\{\left|\alpha_{i}\right|: i \in \mathbb{N}_{N-1}\right\} .
$$

For a fixed base function $b$ and partition $\Delta$, from (2.3), it follows that $\alpha$-fractal function $f^{\alpha}$ converges uniformly to $f \in \mathcal{C}(I)$ only if $|\alpha|_{\infty} \rightarrow 0$.

\section{New class of $\alpha$-fractal approximation and constrained approximation}

In this section, we construct $\alpha$-fractal functions that converge to the original function without any condition on the scaling factors and study constrained approximation by this new class of fractal functions.

Let $b=b_{n}(f)$ for all $n \in \mathbb{N}$. In this case, $\left\{f_{n}^{\alpha}\right\}_{n=1}^{\infty}$ is called a sequence of $\alpha$-fractal functions of $f \in \mathcal{C}(I)$, and

$$
f_{n}^{\alpha}(x)=\alpha_{i} f_{n}^{\alpha}\left(L_{i}^{-1}(x)\right)+f(x)-\alpha_{i} b_{n}(f)\left(L_{i}^{-1}(x)\right), x \in I_{i}, \quad i \in \mathbb{N}_{N-1} .
$$

From the construction of fractal functions (see previous section), it can be verified that for every $n \in \mathbb{N}$, the $\alpha$-fractal function $f_{n}^{\alpha}$, of $f \in \mathcal{C}(I)$ is corresponding to the IFS defined by

$$
\mathcal{I}_{n}=\left\{I \times \mathbb{R},\left(L_{i}(x), F_{n, i}(x, y)\right), i \in \mathbb{N}_{N-1}\right\},
$$

where $F_{n, i}(x, y)=\alpha_{i} y+f\left(L_{i}(x)\right)-\alpha_{i} b_{n}(f)(x)$. The following lemma addresses the convergence of the sequence $\left\{f_{n}^{\alpha}\right\}_{n=1}^{\infty}$ towards $f \in \mathcal{C}(I)$.

Lemma 3.1. Let $\mathcal{C}(I)$ be endowed with $\|f\|_{\infty}=\sup \{|f(x)|: x \in I\}$. Let $f \in \mathcal{C}(I)$. Let $\Delta=$ $\left\{x_{1}, x_{2}, \ldots, x_{N}\right\}$ be a partition of $I=\left[x_{1}, x_{N}\right]$ satisfying $x_{1}<x_{2}<\cdots<x_{N}$. Then, for every scaling vector $\alpha=\left(\alpha_{1}, \alpha_{2}, \ldots, \alpha_{N-1}\right)$, the sequence $\left\{\mathcal{I}_{n}\right\}_{n=1}^{\infty}$ of IFSs determine a sequence $\left\{f_{n}^{\alpha}\right\}_{n=1}^{\infty}$ of $\alpha$-fractal functions that converges uniformly to $f$. 
Proof. From (3.4), it is easy to deduce that

$$
\begin{aligned}
\left\|f_{n}^{\alpha}-f\right\|_{\infty} & \leq|\alpha|_{\infty}\left\|f_{n}^{\alpha}-b_{n}(f)\right\|_{\infty} \\
& \leq|\alpha|_{\infty}\left[\left\|f_{n}^{\alpha}-f\right\|_{\infty}+\left\|f-b_{n}(f)\right\|_{\infty}\right]
\end{aligned}
$$

Hence we obtain

$$
\left\|f_{n}^{\alpha}-f\right\|_{\infty} \leq \frac{|\alpha|_{\infty}}{1-|\alpha|_{\infty}}\left\|f-b_{n}(f)\right\|_{\infty} .
$$

Since the sequence $\left\{b_{n}(f)\right\}_{n=1}^{\infty}$ converges to $f$ uniformly, from (3.6) we can see that $\lim _{n \rightarrow \infty} f_{n}^{\alpha}=f$, and hence the stated result follows.

The following remark follows from Remark 2.1 and Lemma 3.1.

Remark 3.1. Let $\Delta=\left\{x_{1}, x_{2}, \ldots, x_{N}\right\}$ be a partition of $I=\left[x_{1}, x_{N}\right]$ satisfying $x_{1}<x_{2}<\cdots<x_{N}$ and $\alpha=\left(\alpha_{1}, \alpha_{2}, \ldots, \alpha_{N-1}\right)$. If all the $\alpha$-fractal functions in the sequence $\left\{f_{n}^{\alpha}\right\}_{n=1}^{\infty}$ are obtained with the same choice of the scaling factors $\alpha_{i}, i=1,2, \ldots, N-1$, which satisfy the condition $\sum_{i=1}^{N-1}\left|\alpha_{i}\right|>1$, then all the $\alpha$-fractal functions in the sequence $\left\{f_{n}^{\alpha}\right\}_{n=1}^{\infty}$ are nowhere differentiable and $\lim _{n \rightarrow \infty} f_{n}^{\alpha}=f$.

Theorem 3.1. Let $\mathcal{C}(I)$ be endowed with $\|.\|_{\infty}$. Let $\theta_{\epsilon}^{\dagger}=\max \left\{\left\|b_{1}\right\|_{\infty^{*}},\left\|b_{2}\right\|_{\infty^{*}}, \ldots\left\|b_{N_{0}}\right\|_{\infty^{*}}, \epsilon+1\right\}$. For every $n \in \mathbb{N}$, the $\alpha$-operator $\mathcal{F}_{n}^{\alpha}: \mathcal{C}(I) \rightarrow \mathcal{C}(I)$ defined by $\mathcal{F}_{n}^{\alpha}(f)=f_{n}^{\alpha}$ is linear and bounded. Futher, if $|\alpha|_{\infty}<\frac{1}{\theta_{\epsilon}^{\dagger}}$, then $\mathcal{F}_{n}^{\alpha}$ is bounded below and not compact.

Proof. Using (3.4), for each $n \in \mathbb{N}$, we have

$$
\begin{aligned}
& f_{n}^{\alpha}(x)=\alpha_{i} f_{n}^{\alpha}\left(L_{i}^{-1}(x)\right)+f(x)-\alpha_{i} b_{n}(f)\left(L_{i}^{-1}(x)\right), x \in I_{i}, \quad i \in \mathbb{N}_{N-1} . \\
& g_{n}^{\alpha}(x)=\alpha_{i} g_{n}^{\alpha}\left(L_{i}^{-1}(x)\right)+g(x)-\alpha_{i} b_{n}(g)\left(L_{i}^{-1}(x)\right), x \in I_{i}, \quad i \in \mathbb{N}_{N-1} .
\end{aligned}
$$

Multiplying the first equation by $\beta^{*}$ and the second equation by $\gamma^{*}$, and the uniqueness of the solution of the fixed point equation defining the FIF gives:

$$
\left(\beta^{*} f+\gamma^{*} g\right)_{n}^{\alpha}=\beta^{*} f_{n}^{\alpha}+\gamma^{*} g_{n}^{\alpha} \forall \beta^{*}, \gamma^{*} \in \mathbb{R} .
$$

Hence, $\mathcal{F}_{n}^{\alpha}$ is linear. Using (3.6), we get

$$
\left\|f_{n}^{\alpha}\right\|_{\infty} \leq\|f\|_{\infty}+\frac{|\alpha|_{\infty}}{1-|\alpha|_{\infty}}\left\|I_{d}-b_{n}\right\|_{\infty^{*}}\|f\|_{\infty}
$$

where $I_{d}$ is the identity operator and $\|\cdot\|_{\infty^{*}}$ is the operator norm induced by $\|.\|_{\infty}$. Since the sequence $\left\{b_{n}(f)\right\}_{n=1}^{\infty}$ converges to $f$ uniformly, we can see that $\left\|I_{d}-b_{n}\right\|_{\infty^{*}} \rightarrow 0$ as $n \rightarrow \infty$. Therefore, for given $\epsilon>0$, there exists $N_{0} \in \mathbb{N}$ such that

$$
\left\|I_{d}-b_{n}\right\|_{\infty^{*}}<\epsilon \forall n>N_{0} .
$$

Let $\theta_{\epsilon}=\max \left\{\left\|I_{d}-b_{1}\right\|_{\infty^{*}},\left\|I_{d}-b_{2}\right\|_{\infty^{*}}, \ldots\left\|I_{d}-b_{N_{0}}\right\|_{\infty^{*}}, \epsilon\right\}$. Then from (3.7), we get

$$
\left\|f_{n}^{\alpha}\right\|_{\infty} \leq\left(1+\frac{|\alpha|_{\infty} \theta_{\epsilon}}{1-|\alpha|_{\infty}}\right)\|f\|_{\infty} .
$$

Now, using the definition of $\mathcal{F}_{n}^{\alpha}$ in the above inequality, it follows that $\mathcal{F}_{n}^{\alpha}$ is bounded.

Now, in what follows we show that $\mathcal{F}_{n}^{\alpha}$ is not compact. From (3.4), it is easy to deduce that

$$
\left\|f_{n}^{\alpha}-f\right\|_{\infty} \leq|\alpha|_{\infty}\left\|f_{n}^{\alpha}-b_{n}(f)\right\|_{\infty} \Longrightarrow\|f\|_{\infty}-\left\|f_{n}^{\alpha}\right\|_{\infty} \leq|\alpha|_{\infty}\left[\left\|f_{n}^{\alpha}\right\|_{\infty}+\left\|b_{n}(f)\right\|_{\infty}\right] .
$$


From (5.22), we can see that $\left\|b_{n}\right\|_{\infty^{*}}<\theta_{\epsilon}^{\dagger}$ for all $n \in \mathbb{N}$. Hence, further simplification of the previous inequality gives

$$
\left\|\mathcal{F}_{n}^{\alpha}(f)\right\|_{\infty^{*}}=\left\|f_{n}^{\alpha}\right\|_{\infty} \geq \frac{\|f\|_{\infty}\left[1-|\alpha|_{\infty} \theta_{\epsilon}^{\dagger}\right]}{1+|\alpha|_{\infty}}
$$

Now it is plain to see that $\mathcal{F}_{n}^{\alpha}$ is bounded below if $|\alpha|_{\infty}<\frac{1}{\theta_{\epsilon}^{\dagger}}$. Since $\mathcal{F}_{n}^{\alpha}$ is bounded below, it is easy to observe that $\mathcal{F}_{n}^{\alpha}: \mathcal{C}(I) \rightarrow \mathcal{C}(I)$ is injective. Since $\left\{1, x, x^{2}, \ldots,\right\}$ is linearly independent subset of $\mathcal{P}(I)$ the space of all polynomials defined on $I$, and $\mathcal{F}_{n}^{\alpha}$ is injective, the set $\left\{1^{\alpha}, x_{n}^{\alpha},\left(x^{2}\right)_{n}^{\alpha}, \ldots,\right\}$ is linearly independent. Consequently, $\mathcal{P}^{\alpha}(I)=\mathcal{F}_{n}^{\alpha}(\mathcal{P}(I))$ is infinite dimensional, and hence $\mathcal{F}_{n}^{\alpha}(\mathcal{C}(I))$ is infinite dimensional. Since $\mathcal{F}_{n}^{\alpha}$ is bounded below, it follows that $\left(\mathcal{F}_{n}^{\alpha}\right)^{-1}: \mathcal{F}_{n}^{\alpha}(\mathcal{C}(I)) \rightarrow \mathcal{C}(I)$ is continuous. Without loss of generality assume that $\mathcal{F}_{n}^{\alpha}$ is compact. From the operator theory, we can see that the identity operator $I_{d}=\mathcal{F}_{n}^{\alpha}\left(\mathcal{F}_{n}^{\alpha}\right)^{-1}: \mathcal{F}_{n}^{\alpha}(\mathcal{C}(I)) \rightarrow \mathcal{C}(I)$ is compact. This implies that dimension of $\mathcal{C}(I)$ is finite and is a contraction. Hence, the result follows.

The above theorem can be extended to the space of $p$-integrable functions, for details, see [19].

\subsection{Constrained approximation by fractal functions}

Theorem 3.2. Let $f \in \mathcal{C}(I)$. Let $\Delta=\left\{x_{1}, x_{2}, \ldots, x_{N}\right\}$ be a partition of $I=\left[x_{1}, x_{N}\right]$ satisfying $x_{1}<$ $x_{2}<\cdots<x_{N}$. Then, for every scaling vector $\alpha=\left(\alpha_{1}, \alpha_{2}, \ldots, \alpha_{N-1}\right)$, there exists a sequence $\left\{g_{n}^{\alpha}\right\}_{n=1}^{\infty}$ of fractal functions that converges uniformly to $f$ such that $g_{n}^{\alpha}(x) \geq f(x)$ for all $x \in I$ and $n \in \mathbb{N}$. Further, if all the $\alpha$-fractal functions in the sequence $\left\{g_{n}^{\alpha}(x)\right\}_{n=1}^{\infty}$ are constructed with the same choice of the scaling factors $\alpha_{i}, i=1,2, \ldots, N-1$, which satisfy the condition $\sum_{i=1}^{N-1}\left|\alpha_{i}\right|>1$, then all the fractal functions in the sequence $\left\{g_{n}^{\alpha}(x)\right\}_{n=1}^{\infty}$ are nowhere differentiable, $g_{n}^{\alpha}(x) \geq f(x)$ for all $x \in I$, and $\lim _{n \rightarrow \infty} g_{n}^{\alpha}=f$.

Proof. For given $\epsilon>0, f \in \mathcal{C}(I)$, and scaling vector $\alpha$, Theorem 3.1 ensures the existence of a sequence $\left\{f_{n}^{\alpha}\right\}_{n=1}^{\infty}$ of $\alpha$-fractal functions of $f$ such that

$$
\left\|f_{n}^{\alpha}-f\right\|_{\infty}<\frac{\epsilon}{2} \forall n \geq N_{0} \in \mathbb{N}
$$

Define

$$
g_{n}^{\alpha}(x)=f_{n}^{\alpha}(x)+\frac{\epsilon}{2} \forall x \in I \text { and } n \in \mathbb{N} .
$$

We can observe that

$$
g_{n}^{\alpha}(x)=f_{n}^{\alpha}(x)+\mathbf{1}(x) \frac{\epsilon}{2}=f_{n}^{\alpha}(x)+\mathbf{1}^{\alpha}(x) \frac{\epsilon}{2} \forall x \in I \text { and } n \in \mathbb{N} .
$$

Using the linearity of $\mathcal{F}_{n}^{\alpha}$ in the above equation, we obtain that

$$
g_{n}^{\alpha}(x)=f_{n}^{\alpha}(x)+\mathbf{1}^{\alpha} \frac{\epsilon}{2}=\mathcal{F}_{n}^{\alpha}(f)+\frac{\epsilon}{2} \mathcal{F}_{n}^{\alpha}(\mathbf{1})=\mathcal{F}_{n}^{\alpha}\left(f+1 \frac{\epsilon}{2}\right) \forall x \in I \text { and } n \in \mathbb{N} .
$$

Consequently, $g^{\alpha}$ is a fractal function. Next,

$$
g_{n}^{\alpha}(x)=f_{n}^{\alpha}(x)+\frac{\epsilon}{2}=f(x)+f_{n}^{\alpha}(x)+\frac{\epsilon}{2}-f(x) \geq f(x)+\frac{\epsilon}{2}-\left\|f_{n}^{\alpha}-f\right\|_{\infty} \geq f(x) \forall x \in I,
$$


and

$$
\left\|f-g_{n}^{\alpha}\right\|_{\infty} \leq\left\|f-f_{n}^{\alpha}\right\|_{\infty}+\left\|f_{n}^{\alpha}-g_{n}^{\alpha}\right\|_{\infty}<\epsilon .
$$

It establishes the first part of the theorem. The second part of the theorem follows from the above discussion and Theorem 3.1.

Theorem 3.3. Let $\Phi \in \mathcal{C}(I)$ be such that $\Phi(x) \geq 0$ for all $x \in I(\Phi(x) \leq 0$ for all $x \in I)$. Let $\Delta=\left\{x_{1}, x_{2}, \ldots, x_{N}\right\}$ be a partition of $I=\left[x_{1}, x_{N}\right]$ satisfying $x_{1}<x_{2}<\cdots<x_{N}$. Then, for every scaling vector $\alpha=\left(\alpha_{1}, \alpha_{2}, \ldots, \alpha_{N-1}\right)$, there exists a sequence of positive (negative) fractal functions that converges uniformly to $\Phi$.

Proof. From the Weierstrass theorem, one can show that for $\Phi \in \mathcal{C}(I)$ there exists a polynomial $f$ satisfying $f(x) \geq \Phi(x)$ for all $x \in I$ and $\|\Phi-f\|_{\infty}<\frac{\epsilon}{3}$. For given $\epsilon>0, f \in \mathcal{C}(I)$, and scaling vector $\alpha$, Theorem 3.1 ensures the existence of a sequence $\left\{f_{n}^{\alpha}\right\}_{n=1}^{\infty}$ of $\alpha$-fractal polynomials of $f$ such that

$$
\left\|f_{n}^{\alpha}-f\right\|_{\infty}<\frac{\epsilon}{3} \forall n \geq N_{0} \in \mathbb{N} .
$$

Define

$$
g_{n}^{\alpha}(x)=f_{n}^{\alpha}(x)+\frac{\epsilon}{3} \forall x \in I, n \in \mathbb{N} .
$$

Next,

$$
g_{n}^{\alpha}(x)=f_{n}^{\alpha}(x)+\frac{\epsilon}{3}=f(x)+f_{n}^{\alpha}(x)+\frac{\epsilon}{3}-f(x) \geq f(x)+\frac{\epsilon}{3}-\left\|f_{n}^{\alpha}-f\right\|_{\infty} \geq f(x) \geq \Phi(x) \geq 0 \forall x \in I .
$$

The first part of the proof is completed by noting that

$$
\left\|\Phi-g_{n}^{\alpha}\right\|_{\infty} \leq\|\Phi-f\|_{\infty}+\left\|f-f_{n}^{\alpha}\right\|_{\infty}+\left\|f_{n}^{\alpha}-g_{n}^{\alpha}\right\|_{\infty}<\epsilon .
$$

For second part of the proof, we proceed as follows:

Again from the Weierstrass theorem, one can show that for $\Phi \in \mathcal{C}(I)$ there exists a polynomial $f$ satisfying $f(x) \leq \Phi(x)$ for all $x \in I$ and $\|\Phi-f\|_{\infty}<\frac{\epsilon}{3}$. For given $\epsilon>0, f \in \mathcal{C}(I)$, and scaling vector $\alpha$, Theorem 3.1 ensures the existence of a sequence $\left\{f_{n}^{\alpha}\right\}_{n=1}^{\infty}$ of $\alpha$-fractal polynomials of $f$ such that

$$
\left\|f_{n}^{\alpha}-f\right\|_{\infty}<\frac{\epsilon}{3} \forall n \geq N_{0} \in \mathbb{N} .
$$

Define

$$
h_{n}^{\alpha}(x)=f_{n}^{\alpha}(x)-\frac{\epsilon}{3} \forall x \in I, n \in \mathbb{N} .
$$

Next,

$$
h_{n}^{\alpha}(x)=f_{n}^{\alpha}(x)-\frac{\epsilon}{3}=f(x)+f_{n}^{\alpha}(x)-\frac{\epsilon}{3}-f(x) \leq f(x)-\frac{\epsilon}{3}-\left\|f_{n}^{\alpha}-f\right\|_{\infty} \leq f(x) \leq \Phi(x) \forall x \in I .
$$

The second part of the proof is completed by noting that

$$
\left\|\Phi-h_{n}^{\alpha}\right\|_{\infty} \leq\|\Phi-f\|_{\infty}+\left\|f-f_{n}^{\alpha}\right\|_{\infty}+\left\|f_{n}^{\alpha}-h_{n}^{\alpha}\right\|_{\infty}<\epsilon .
$$

Remark 3.2. The Theorem 3.3 can be extended to Bernstein polynomials. 


\section{Approximation by fractal polynomials}

Let $\mathcal{P}_{n}(I)$ be the space of all polynomials of degree less than or equal to $n \in \mathbb{N}$ and $\mathcal{P}(I)$ be the space of all polynomials defined on $I$. Since the fractal operator $\mathcal{F}_{n}^{\alpha}: \mathcal{C}(I) \rightarrow \mathcal{C}(I)$ is linear and bounded, the image set $\mathcal{F}_{n}^{\alpha}(\mathcal{P}(I))=\mathcal{P}^{\alpha}(I)$ is the set of all $\alpha$-fractal polynomials.

\subsection{Existence of Schauder basis of fractal polynomials for $\mathcal{C}(I)$}

Definition 4.1. A Schauder basis for a normed linear space $E$ is a sequence $\left\{\phi_{k}\right\}_{k=1}^{\infty}$ such that each element $f \in E$ is written uniquely as $f=\sum_{k=1}^{\infty} d_{k} \phi_{k}$.

Theorem 4.1. There exists a Schauder basis of fractal polynomials for $\mathcal{C}(I)$.

Proof. Let $\left\{\phi_{k}\right\}_{k=1}^{\infty}$ be a Schauder basis of polynomials for $\mathcal{C}(I)$. Hence, for all $f \in \mathcal{C}(I)$, we have

$$
f=\sum_{k=1}^{\infty} d_{k} \phi_{k}
$$

Let $\Delta=\left\{x_{1}, x_{2}, \ldots, x_{N}\right\}$ be a partition of $I=\left[x_{1}, x_{N}\right]$ satisfying $x_{1}<x_{2}<\cdots<x_{N}$, and scaling vector $\alpha=\left(\alpha_{1}, \alpha_{2}, \ldots, \alpha_{N-1}\right)$, By Theorem 3.1, for each polynomial $\phi_{k}$ and any scaling vector $\alpha$, there exists a fractal polynomial $\left(\phi_{k}\right)_{n_{k}}^{\alpha}$ such that

$$
\left\|\phi_{k}-\left(\phi_{k}\right)_{n_{k}}^{\alpha}\right\|_{\infty}<\frac{1}{\left|d_{k}(f)\right| 2^{k}}, \text { provided }\left|d_{k}(f)\right| \neq 0 .
$$

Let us define the series

$$
\sum_{k=1}^{\infty} d_{k}(f)\left(\phi_{k}\right)_{n_{k}}^{\alpha}
$$

where $d_{k}(f)$ are the coefficients of $f$ with respect to the Schauder basis $\left\{\phi_{k}\right\}_{k=1}^{\infty}$. Now, we are going to prove the convergence of the above series. Let $u, v \in \mathbb{N}$ such that $u>v$ and $S_{u}=\sum_{k=1}^{u} d_{k}(f)\left(\phi_{k}\right)_{n_{k}}^{\alpha}$. Then

$$
\begin{aligned}
\left\|S_{u}-S_{v}\right\|_{\infty} & =\left\|\sum_{k=v+1}^{u} d_{k}(f)\left(\phi_{k}\right)_{n_{k}}^{\alpha}\right\|_{\infty} \\
& \leq \sum_{k=v+1}^{u}\left|d_{k}(f)\right|\left\|\left(\phi_{k}\right)_{n_{k}}^{\alpha}-\phi_{k}\right\|_{\infty}+\left\|\sum_{k=v+1}^{u} d_{k}(f) \phi_{k}\right\|_{\infty}
\end{aligned}
$$

Using (4.10) in (4.11), we get

$$
\begin{aligned}
\left\|S_{u}-S_{v}\right\|_{\infty} & \leq \sum_{k=v+1}^{u} \frac{1}{2^{k}}+\left\|\sum_{k=v+1}^{u} d_{k}(f) \phi_{k}\right\|_{\infty} \\
& =\sum_{k=1}^{u} \frac{1}{2^{k}}-\sum_{k=1}^{v} \frac{1}{2^{k}}+\left\|\sum_{k=1}^{u} d_{k}(f) \phi_{k}-\sum_{k=1}^{v} d_{k}(f) \phi_{k}\right\|_{\infty}
\end{aligned}
$$


Since $\sum_{k=1}^{\infty} \frac{1}{2^{k}}$ and $\sum_{k=1}^{\infty} d_{k}(f) \phi_{k}$ are convergent, for given $\epsilon>0$, there exist $N_{1}, N_{2} \in \mathbb{N}$ such that

$$
\begin{aligned}
& \sum_{k=1}^{u} \frac{1}{2^{k}}-\sum_{k=1}^{v} \frac{1}{2^{k}}<\frac{\epsilon}{2} \forall u, v>N_{1}, \\
& \left\|\sum_{k=1}^{u} d_{k}(f) \phi_{k}-\sum_{k=1}^{v} d_{k}(f) \phi_{k}\right\|_{\infty}<\frac{\epsilon}{2} \forall u, v>N_{2} .
\end{aligned}
$$

Using (4.13) in (4.12), we get

$$
\left\|S_{u}-S_{v}\right\|_{\infty}<\epsilon \forall u, v>N_{3}=\max \left\{N_{1}, N_{2}\right\} .
$$

Therefore, $\left\{S_{u}\right\}_{u=1}^{\infty}$ is a Cauchy sequence, and hence $\sum_{k=1}^{\infty} d_{k}(f)\left(\phi_{k}\right)_{n_{k}}^{\alpha}$ is convergent. Next,

$$
\left\|\sum_{k=1}^{\infty} d_{k}(f)\left(\phi_{k}\right)_{n_{k}}^{\alpha}-f\right\|_{\infty}=\left\|\sum_{k=1}^{\infty} d_{k}(f)\left(\phi_{k}\right)_{n_{k}}^{\alpha}-\sum_{k=1}^{\infty} d_{k}(f) \phi_{k}\right\|_{\infty} \leq \sum_{k=1}^{\infty}\left|d_{k}(f)\right|\left\|\left(\phi_{k}\right)_{n_{k}}^{\alpha}-\phi_{k}\right\|_{\infty} .
$$

Using (4.10) in the above inequality, we conclude that the series $\sum_{k=1}^{\infty} d_{k}(f)\left(\phi_{k}\right)_{n_{k}}^{\alpha}$ converges to $f$, that is

$$
f=\sum_{k=1}^{\infty} d_{k}(f)\left(\phi_{k}\right)_{n_{k}}^{\alpha}
$$

In what follows we show that representation of $f$ in (4.14) is unique. For this purpose let

$$
f=\sum_{k=1}^{\infty} e_{k}(f)\left(\phi_{k}\right)_{n_{k}}^{\alpha}
$$

We write (4.14) for the function $\phi_{k}$ as follows:

$$
\phi_{k}=\left(\phi_{k}\right)_{n_{k}}^{\alpha}
$$

Substituting (4.16) in (4.15), we get $f=\sum_{k=1}^{\infty} e_{k}(f) \phi_{k}$. Now, using the fact that $\left\{\phi_{k}\right\}_{k=1}^{\infty}$ is Schauder basis, it follows that $e_{k}(f)=d_{k}(f)$ for all $k \in \mathbb{N}$. Therefore, the representation of $f$ in (4.14) is unique. From the above discussion, we conclude that $\left\{\left(\phi_{k}\right)_{n_{k}}^{\alpha}\right\}_{k=1}^{\infty}$ is a Schauder basis for $\mathcal{C}(I)$.

Remark 4.1. Since (4.10) is true for any scaling vector $\alpha$, if we choose the scaling vector $\alpha$ so that its components obey the condition $\sum_{i=1}^{N-1}\left|\alpha_{i}\right|>1$, then all the fractal polynomials in the Schauder basis $\left\{\left(\phi_{k}\right)_{n_{k}}^{\alpha}\right\}_{k=1}^{\infty}$ are nowhere differentiable.

\section{Mean square fractal approximation of complex-valued functions}

Let $L^{2}(I, \mathbb{C})=\left\{h \mid h: I \rightarrow \mathbb{C}\right.$ and $\left.\|h\|_{L^{2}}<\infty\right\}$. In this section, we show that for $h \in L^{2}(I, \mathbb{C})$, there exists a sequence of complex-valued fractal functions that converges to $h$.

Theorem 5.1. Let $f \in \mathcal{C}\left[x_{1}, x_{N}\right]$ and $\|f\|_{L^{2}}=\left(\int_{x_{1}}^{x_{N}}|f(x)|^{2} d x\right)^{\frac{1}{2}}$. Let $b_{n}: \mathcal{C}(I) \rightarrow \mathcal{C}(I)$ be a bounded and linear operator such that for every $f \in \mathcal{C}(I), b_{n}(f)\left(x_{1}\right)=f\left(x_{1}\right), b_{n}(f)\left(x_{N}\right)=f\left(x_{N}\right)$, and $\| b_{n}(f)-$ $f \|_{L^{2}} \rightarrow 0$ uniformly as $n \rightarrow \infty$. Then the following inequalities are true:

$$
\left\|f_{n}^{\alpha}-f\right\|_{L^{2}} \leq \frac{|\alpha|_{\infty}}{1-|\alpha|_{\infty}}\left\|f-b_{n}(f)\right\|_{L^{2}}
$$




$$
\begin{gathered}
\left\|\mathcal{F}_{n}^{\alpha}-I_{d}\right\|_{2} \leq \frac{|\alpha|_{\infty}}{1-|\alpha|_{\infty}} \Theta_{\epsilon}, \\
\left\|\mathcal{F}_{n}^{\alpha}\right\|_{2} \leq 1+\frac{|\alpha|_{\infty}}{1-|\alpha|_{\infty}} \Theta_{\epsilon},
\end{gathered}
$$

where $I_{d}$ is the identity operator and $\|\cdot\|_{2}$ is the operator norm induced by $\|\cdot\|_{L^{2}}, \Theta_{\epsilon}=\max \left\{\| I_{d}-\right.$ $\left.b_{1}\left\|_{2},\right\| I_{d}-b_{2}\left\|_{2}, \ldots\right\| I_{d}-b_{N_{0}} \|_{2}, \epsilon\right\}, \epsilon>0$.

Proof. From (3.4), it is easy to deduce that

$$
\left\|f_{n}^{\alpha}-f\right\|_{L^{2}}^{2}=\sum_{i=1}^{N-1} \int_{x_{i}}^{x_{i+1}}\left|\alpha_{i}\right|^{2}\left|f_{n}^{\alpha}\left(L_{i}^{-1}(x)\right)-b_{n}(f)\left(L_{i}^{-1}(x)\right)\right|^{2} d x .
$$

Let $L_{i}^{-1}(x)=t$. Then $d x=a_{i} d t$ and $t \in\left[x_{1}, x_{N}\right]$. Therefore,

$$
\left\|f_{n}^{\alpha}-f\right\|_{L^{2}}^{2}=\sum_{i=1}^{N-1} a_{i}\left|\alpha_{i}\right|^{2} \int_{x_{1}}^{x_{N}}\left|f_{n}^{\alpha}(t)-b_{n}(f)(t)\right|^{2} d t \leq|\alpha|_{\infty}^{2}\left\|f_{n}^{\alpha}-b_{n}(f)\right\|_{L^{2}}^{2} \sum_{i=1}^{N-1} a_{i} .
$$

But $\sum_{i=1}^{N-1} a_{i}=\sum_{i=1}^{N-1} \frac{x_{i+1}-x_{i}}{x_{N}-x_{1}}=1$. Therefore, the previous inequality leads to

$$
\left\|f_{n}^{\alpha}-f\right\|_{L^{2}} \leq|\alpha|_{\infty}\left\|f_{n}^{\alpha}-b_{n}(f)\right\|_{L^{2}} \leq|\alpha|_{\infty}\left[\left\|f_{n}^{\alpha}-f\right\|_{L^{2}}+\left\|f-b_{n}(f)\right\|_{L^{2}}\right] .
$$

Simplifying the above inequality, we get

$$
\left\|f_{n}^{\alpha}-f\right\|_{L^{2}} \leq \frac{|\alpha|_{\infty}}{1-|\alpha|_{\infty}}\left\|f-b_{n}(f)\right\|_{L^{2}} \Rightarrow\left\|f_{n}^{\alpha}-f\right\|_{L^{2}} \leq \frac{|\alpha|_{\infty}}{1-|\alpha|_{\infty}}\|f\|_{L^{2}}\left\|I_{d}-b_{n}\right\|_{2} .
$$

Since $\left\|f-b_{n}(f)\right\|_{L^{2}} \rightarrow 0$ as $n \rightarrow \infty$, for given $\epsilon>0$, there exists $N_{0} \in \mathbb{N}$ such that

$$
\left\|f-b_{n}(f)\right\|_{L^{2}}<\epsilon \forall n>N_{0} \Rightarrow\left\|I_{d}-b_{n}\right\|_{2}<\epsilon \forall n>N_{0} .
$$

Then, from (5.22), we get

$$
\left\|I_{d}-b_{n}\right\|_{2}<\Theta_{\epsilon} \forall n \in \mathbb{N} .
$$

Using (5.23) in (5.21) and simplifying (5.21), we get

$$
\left\|f_{n}^{\alpha}-f\right\|_{L^{2}} \leq \frac{|\alpha|_{\infty}}{1-|\alpha|_{\infty}} \Theta_{\epsilon}\|f\|_{L^{2}}
$$

Now, using the definition of $\mathcal{F}_{n}^{\alpha}$, the above inequality yields (5.18). Next, by rewriting (5.18) as

$$
\left\|\mathcal{F}_{n}^{\alpha}\right\|_{2}-\left\|I_{d}\right\|_{2} \leq\left\|\mathcal{F}_{n}^{\alpha}-I_{d}\right\|_{L^{2}} \leq \frac{|\alpha|_{\infty}}{1-|\alpha|_{\infty}} \Theta_{\epsilon}
$$

we obtain (5.19).

Theorem 5.2. There exists a linear and bounded operator $\mathcal{F}_{n}^{* \alpha}$ on the Hilbert space $L^{2}(I, \mathbb{C})$ such that restriction of $\mathcal{F}_{n}^{* \alpha}$ on $\mathcal{C}(I)$ is $\mathcal{F}_{n}^{\alpha}$ and $\left\|\mathcal{F}_{n}^{* \alpha}\right\|_{2}=\left\|\mathcal{F}_{n}^{\alpha}\right\|_{2}$.

Proof. We define $\alpha$-fractal operator $\mathcal{F}_{n, \mathbb{C}}^{\alpha}$ on the space $\mathcal{C}(I, \mathbb{C})$ of complex-valued continuous functions defined on the interval $I=\left[x_{1}, x_{N}\right]$ as follows:

$$
\mathcal{F}_{n, \mathbb{C}}^{\alpha}(f+i g):=\mathcal{F}_{n}^{\alpha}(f)+i \mathcal{F}_{n}^{\alpha}(g) \forall f, g \in \mathcal{C}(I) .
$$


In the construction of $\mathcal{F}_{n}^{\alpha}(f)$ and $\mathcal{F}_{n}^{\alpha}(g)$, we take $b_{n}(f)$ and $b_{n}(g)$ as base functions respectively, where $b_{n}$ is a bounded and linear operator on $\mathcal{C}(I)$ such that for every $\tau \in \mathcal{C}(I), b_{n}(\tau)\left(x_{1}\right)=\tau\left(x_{1}\right), b_{n}(\tau)\left(x_{N}\right)=$ $\tau\left(x_{N}\right)$, and $\left\|b_{n}(\tau)-\tau\right\|_{L^{2}} \rightarrow 0$ uniformly as $n \rightarrow \infty$.

Since $\mathcal{F}_{n}^{\alpha}$ is linear, it follows that $\mathcal{F}_{n, \mathbb{C}}^{\alpha}$ is linear. Next, it is easy to see from (5.26) that

$$
\left\|\mathcal{F}_{n, \mathbb{C}}^{\alpha}(f+i g)\right\|_{L^{2}}^{2}=\int_{x_{1}}^{x_{N}}\left|\mathcal{F}_{n}^{\alpha}(f)\right|^{2} d x+\int_{x_{1}}^{x_{N}}\left|\mathcal{F}_{n}^{\alpha}(g)\right|^{2} d x=\left\|\mathcal{F}_{n}^{\alpha}(f)\right\|_{L^{2}}^{2}+\left\|\mathcal{F}_{n}^{\alpha}(g)\right\|_{L^{2}}^{2} .
$$

Now, it follows that

$$
\left\|\mathcal{F}_{n, \mathbb{C}}^{\alpha}(f+i g)\right\|_{L^{2}}^{2} \leq\left\|\mathcal{F}_{n}^{\alpha}\right\|_{L^{2}}^{2}\left[\|f+i g\|_{L^{2}}^{2}\right] \forall f+i g \in \mathcal{C}(I, \mathbb{C}),
$$

and

$$
\left\|\mathcal{F}_{n, \mathbb{C}}^{\alpha}\right\|_{L^{2}} \leq\left\|\mathcal{F}_{n}^{\alpha}\right\|_{L^{2}}
$$

Hence $\mathcal{F}_{n, \mathbb{C}}^{\alpha}: \mathcal{C}(I, \mathbb{C}) \rightarrow \mathcal{C}(I, \mathbb{C}) \subset L^{2}(I, \mathbb{C})$ is bounded linear operator. It is known that $\mathcal{C}(I, \mathbb{C})$ is dense in $L^{2}(I, \mathbb{C})$. Therefore, using [20], there exists a $\alpha$-operator $\mathcal{F}_{n}^{* \alpha}: L^{2}(I, \mathbb{C}) \rightarrow L^{2}(I, \mathbb{C})$ such that

$$
\left\|\mathcal{F}_{n}^{* \alpha}\right\|_{2}=\left\|\mathcal{F}_{n, \mathbb{C}}^{\alpha}\right\|_{2} \leq\left\|\mathcal{F}_{n}^{\alpha}\right\|_{2} .
$$

For $f \in \mathcal{C}(I)$, we can notice that

$$
\mathcal{F}_{n}^{* \alpha}(f)=\mathcal{F}_{n, \mathbb{C}}^{\alpha}(f)=\mathcal{F}_{n}^{\alpha}(f)
$$

Then,

$$
\begin{aligned}
\left\|\mathcal{F}_{n}^{\alpha}\right\|_{2} & =\sup \left\{\left\|\mathcal{F}_{n}^{\alpha}(f)\right\|_{L^{2}} ; f \in \mathcal{C}(I),\|f\|_{L^{2}}=1\right\}, \\
& =\sup \left\{\left\|\mathcal{F}_{n, \mathbb{C}}^{\alpha}(f)\right\|_{L^{2}} ; f \in \mathcal{C}(I),\|f\|_{L^{2}}=1\right\} \leq\left\|\mathcal{F}_{n}^{* \alpha}\right\|_{2} .
\end{aligned}
$$

From (5.27) and (5.29), we get

$$
\left\|\mathcal{F}_{n}^{* \alpha}\right\|_{2}=\left\|\mathcal{F}_{n, \mathbb{C}}^{\alpha}\right\|_{2}=\left\|\mathcal{F}_{n}^{\alpha}\right\|_{2}
$$

\subsection{Construction of complex fractal approximants}

Lemma 5.1. For all $n \in \mathbb{N}$, let $b_{n}: \mathcal{C}(I) \rightarrow \mathcal{C}(I)$ be bounded and linear operator such that for every $f \in \mathcal{C}(I), b_{n}(f)\left(x_{1}\right)=f\left(x_{1}\right), b_{n}(f)\left(x_{N}\right)=f\left(x_{N}\right)$, and $\left\|b_{n}(f)-f\right\|_{L^{2}} \rightarrow 0$ as $n \rightarrow \infty$. For all $h \in L^{2}(I, \mathbb{C})$,

$$
\left\|h_{n}^{\alpha}-h\right\|_{L^{2}} \leq|\alpha|_{\infty}\left\|h_{n}^{\alpha}-b_{n}^{*}(h)\right\|_{L^{2}} \forall n \in \mathbb{N},
$$

where $b_{n}^{*}$ is bounded and linear operator on $L^{2}(I, \mathbb{C})$ given in the proof.

Proof. First we prove the result for all $h=f+i g \in \mathcal{C}(I, \mathbb{C})$. Let $b_{n, \mathbb{C}}: \mathcal{C}(I, \mathbb{C}) \rightarrow \mathcal{C}(I, \mathbb{C})$ be defined by

$$
b_{n, \mathbb{C}}(h)=b_{n}(f)+i b_{n}(g) \forall h=f+i g \in \mathcal{C}(I, \mathbb{C}) .
$$


Using Theorem 5.2, we can see that $\left\|b_{n, \mathbb{C}}\right\|_{L^{2}} \leq\left\|b_{n}\right\|_{L^{2}}$ for all $n \in \mathbb{N}$. Hence, using [20], there exists $b_{n}^{*}: L^{2}(I, \mathbb{C}) \rightarrow L^{2}(I, \mathbb{C})$ such that

$$
\left\|b_{n, \mathbb{C}}\right\|_{L^{2}}=\left\|b_{n}^{*}\right\|_{L^{2}}, b_{n, \mathbb{C}}(h)=b_{n}^{*}(h) \forall h \in \mathcal{C}(I, \mathbb{C}) .
$$

We rewrite (5.26) in the following way:

$$
h_{n}^{\alpha}:=f_{n}^{\alpha}+i g_{n}^{\alpha} \quad \forall h=f+i g \in \mathcal{C}(I, \mathbb{C}) \forall n \in \mathbb{N} .
$$

In the construction of $f_{n}^{\alpha}$ and $g_{n}^{\alpha}$, we take $b_{n}(f)$ and $b_{n}(g)$ as base functions respectively. Using (5.32), we obtain that

$$
\left\|h_{n}^{\alpha}-h\right\|_{L^{2}}^{2}=\left\|f_{n}^{\alpha}-f\right\|_{L^{2}}^{2}+\left\|g_{n}^{\alpha}-g\right\|_{L^{2}}^{2} .
$$

Using (5.20) for $f$ and $g$, we get the following:

$$
\begin{aligned}
& \left\|f_{n}^{\alpha}-f\right\|_{L^{2}} \leq|\alpha|_{\infty}\left\|f_{n}^{\alpha}-b_{n}(f)\right\|_{L^{2}}, \\
& \left\|g_{n}^{\alpha}-g\right\|_{L^{2}} \leq|\alpha|_{\infty}\left\|g_{n}^{\alpha}-b_{n}(g)\right\|_{L^{2}} .
\end{aligned}
$$

Using (5.34) in (5.33) and further simplifying, we obtain

$$
\left\|h_{n}^{\alpha}-h\right\|_{L^{2}}^{2} \leq|\alpha|_{\infty}^{2}\left(\left\|h_{n}^{\alpha}-b_{n, \mathbb{C}}(h)\right\|_{L^{2}}^{2}\right) .
$$

Using (5.31) in (5.35), we get the result for all $h=f+i g \in \mathcal{C}(I, \mathbb{C})$. In what follows, we prove the result for $h=f+i g \in L^{2}(I, \mathbb{C})$. For $h \in L^{2}(I, \mathbb{C})$, there exists a sequence $\left\{r_{k}\right\}$ in $\mathcal{C}(I, \mathbb{C})$ such that $\lim _{k \rightarrow \infty} r_{k}=h$ in $L^{2}$-norm. The continuity of $b_{n}^{*}$ implies that

$$
\lim _{k \rightarrow \infty} b_{n}^{*}\left(r_{k}\right)=b_{n}^{*}(h) .
$$

Bearing this in mind, we get the following:

$$
\left\|h_{n}^{\alpha}-h\right\|_{L^{2}}^{2}=\left\|\left(\lim _{k \rightarrow \infty} r_{k}\right)_{n}^{\alpha}-\lim _{k \rightarrow \infty} r_{k}\right\|_{L^{2}}^{2}=\lim _{k \rightarrow \infty}\left\|\left(r_{k}\right)_{n}^{\alpha}-r_{k}\right\|_{L^{2}}^{2}
$$

Using (5.35) in (5.37), we get

$$
\left\|h_{n}^{\alpha}-h\right\|_{L^{2}}^{2} \leq|\alpha|_{\infty_{k}}^{2} \lim _{k \rightarrow \infty}\left\|\left(r_{k}\right)_{n}^{\alpha}-b_{n, \mathbb{C}}\left(r_{k}\right)\right\|_{L^{2}}^{2}
$$

Using (5.31) and (5.36) in (5.38), we obtain

$$
\left\|h_{n}^{\alpha}-h\right\|_{L^{2}}^{2} \leq|\alpha|_{\infty}^{2}\left\|h_{n}^{\alpha}-b_{n}^{*}(h)\right\|_{L^{2}}^{2} .
$$

This completes the proof.

Theorem 5.3. For every $n \in \mathbb{N}$, let $b_{n}: \mathcal{C}(I) \rightarrow \mathcal{C}(I)$ be bounded and linear operator such that for every $f \in \mathcal{C}(I), b_{n}(f)\left(x_{1}\right)=f\left(x_{1}\right), b_{n}(f)\left(x_{N}\right)=f\left(x_{N}\right)$, and $\left\|b_{n}(f)-f\right\|_{L^{2}} \rightarrow 0$ as $n \rightarrow \infty$. Then for all $h \in L^{2}(I, \mathcal{C})$,

$$
\left\|h_{n}^{\alpha}-h\right\|_{L^{2}} \leq \frac{|\alpha|_{\infty}}{1-|\alpha|_{\infty}}\left\|b_{n}-I_{d}\right\|_{2}\|h\|_{L^{2}} \forall n \in \mathbb{N} .
$$

In other words, for every scaling vector $\alpha=\left(\alpha_{1}, \alpha_{2}, \ldots, \alpha_{N-1}\right)$, the sequence $\left\{h_{n}\right\}_{n=1}^{\infty}$ of complex valued functions converges uniformly to $h$ in $L^{2}$-norm. Further, if all the $\alpha$-fractal functions in the sequence $\left\{h_{n}^{\alpha}\right\}_{n=1}^{\infty}$ are constructed with the same choice of the scaling factors $\alpha_{i}, i=1,2, \ldots, N-$ 1 , which satisfy the condition $\sum_{i=1}^{N-1}\left|\alpha_{i}\right|>1$, then the real and imaginary parts of all the $\alpha$-fractal functions in the sequence $\left\{h_{n}^{\alpha}\right\}_{n=1}^{\infty}$ are nowhere differentiable and $\lim _{n \rightarrow \infty} h_{n}^{\alpha}=f$ in $L^{2}$-norm. 
Proof. Using Lemma 5.1,

$$
\left\|h_{n}^{\alpha}-h\right\|_{L^{2}} \leq|\alpha|_{\infty}\left\|h_{n}^{\alpha}-b_{n}^{*}(h)\right\|_{L^{2}} \leq|\alpha|_{\infty}\left(\left\|h_{n}^{\alpha}-h\right\|_{L^{2}}+\left\|h-b_{n}^{*}(h)\right\|_{L^{2}}\right) .
$$

Then,

$$
\left\|h_{n}^{\alpha}-h\right\|_{L^{2}} \leq|\alpha|_{\infty}\left(\left\|h_{n}^{\alpha}-h\right\|_{L^{2}}+\left\|I_{d}-b_{n}^{*}\right\|_{2}\|h\|_{L^{2}}\right) .
$$

Since $b_{n}$ is linear and bounded, it follows that $I_{d}-b_{n}$ is linear and bounded. Now, we define $\left(I_{d}-b_{n}\right)_{\mathbb{C}}$ : $\mathcal{C}(I, \mathbb{C}) \rightarrow \mathcal{C}(I, \mathbb{C})$ as

$$
\left(I_{d}-b_{n}\right)_{\mathbb{C}}(h)=h-b_{n, \mathbb{C}}(h) \forall h \in \mathcal{C}(I, \mathbb{C}) .
$$

It is easy to calculate that

$$
\left\|\left(I_{d}-b_{n}\right)_{\mathbb{C}}\right\|_{L^{2}} \leq\left\|\left(I_{d}-b_{n}\right)\right\|_{L^{2}} .
$$

Using [20], there exists a bounded linear operator $\left(I_{d}-b_{n}\right)^{*}: L^{2}(I, \mathbb{C}) \rightarrow L^{2}(I, \mathbb{C})$ such that

$$
\left(I_{d}-b_{n}\right)^{*}(h)=\left(I_{d}-b_{n}\right)_{\mathbb{C}}(h) \forall h \in \mathcal{C}(I, \mathbb{C}),\left\|\left(I_{d}-b_{n}\right)^{*}\right\|_{L^{2}}=\left\|\left(I_{d}-b_{n}\right)_{\mathbb{C}}\right\|_{L^{2}}
$$

On the lines similar to that of Theorem 5.2, it follows that

$$
\left\|\left(I_{d}-b_{n}\right)^{*}\right\|_{L^{2}}=\left\|\left(I_{d}-b_{n}\right)_{\mathbb{C}}\right\|_{L^{2}}=\left\|I_{d}-b_{n}\right\|_{L^{2}}
$$

Since $\mathcal{C}(I, \mathbb{C})$ is dense in $L^{2}(I, \mathbb{C})$, for $h \in L^{2}(I, \mathbb{C})$ there exists a sequence $\left\{h_{k}\right\}_{k=1}^{\infty}$ in $\mathcal{C}(I, \mathbb{C})$ such that $h_{k} \rightarrow h$ as $k \rightarrow \infty$. Now using the continuity of $\left(I_{d}-b_{n}\right)^{*}$ and (5.42), we get the following: for all $h \in L^{2}(I, \mathbb{C})$,

$$
\left(I_{d}-b_{n}\right)^{*}(h)=\left(I_{d}-b_{n}\right)^{*}\left(\lim _{k \rightarrow \infty} h_{k}\right)=\lim _{k \rightarrow \infty}\left(I_{d}-b_{n}\right)^{*}\left(h_{k}\right)=\lim _{k \rightarrow \infty}\left(I_{d}-b_{n}\right)_{\mathbb{C}}\left(h_{k}\right)=\lim _{k \rightarrow \infty}\left(h_{k}-b_{n, \mathbb{C}} h_{k}\right) .
$$

Using (5.31) and continuity of $b_{n}^{*}$ in (5.44), we obtain

$$
\left(I_{d}-b_{n}\right)^{*}(h)=h-b_{n}^{*}(h) \forall h \in L^{2}(I, \mathbb{C}) .
$$

Finally using (5.43) in (5.45), we get

$$
\left\|\left(I_{d}-b_{n}\right)^{*}\right\|_{L^{2}}=\left\|I_{d}-b_{n}^{*}\right\|_{L^{2}}=\left\|I_{d}-b_{n}\right\|_{L^{2}} .
$$

Using (5.46) in (5.40) we get the first part of the theorem. The second part of the theorem follows from the above discussion and Theorem 3.1 .

\subsection{Examples}

In this section, we provide numerical examples to corroborate our findings. For this purpose, let $f(x)=$ $\sin (\pi x)+\cos (\pi x), x \in[0,1]$. The graph of $f(x)=\sin (\pi x)+\cos (\pi x), x \in[0,1]$ is given in Figure 1(a). The $\alpha$-fractal functions in Figures 1(b)-(f) are generated with respect to the partition $\Delta=\{0,0.1111$, $0.2222,0.3333,0.4444,0.5556,0.6667,0.7778,0.8889,1\}$ of $[0,1]$ and by taking $b_{n}(f)$ as Bernstein polynomial of $f$ on $[0,1]$, i.e.,

$$
b_{n}(f)(x)=B_{n}(f, x)=\sum_{k=0}^{n}\left(\begin{array}{c}
n \\
k
\end{array}\right) x^{k}(1-x)^{n-k} f\left(\frac{k}{n}\right) \forall x \in[0,1], n \in \mathbb{N} .
$$




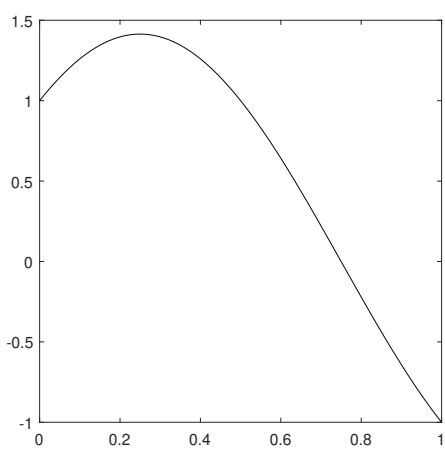

(a): $f(x)=\sin (\pi x)+\cos (\pi x), x \in[0,1]$.

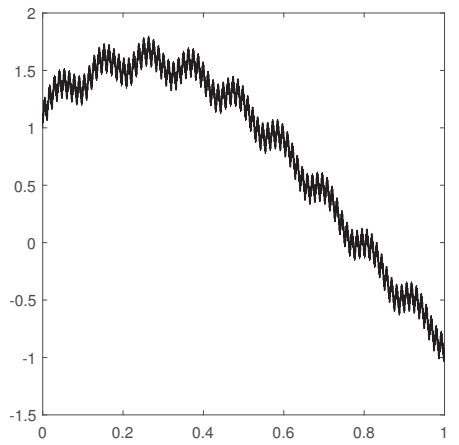

(c): $f_{7}^{\alpha}$ with $\alpha_{i}=0.8, i \in \mathbb{N}_{9}$.

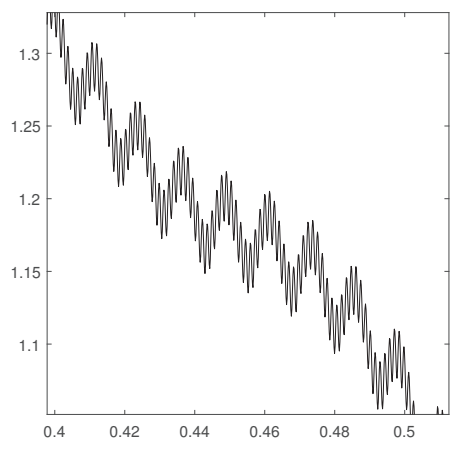

(e): A part of $f_{26}^{\alpha}$ under magnification

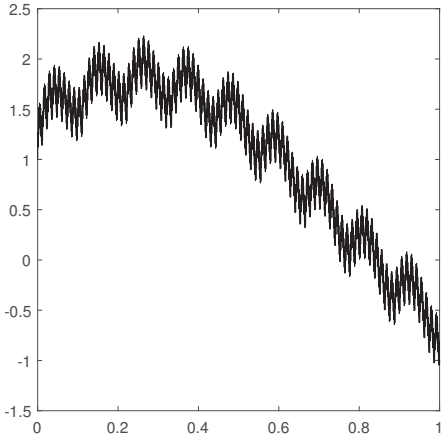

(b): $f_{3}^{\alpha}$ with $\alpha_{i}=0.8, i \in \mathbb{N}_{9}$.

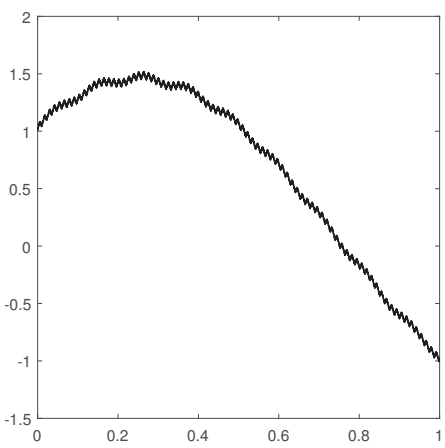

(d): $f_{26}^{\alpha}$ with $\alpha_{i}=0.8, i \in \mathbb{N}_{9}$.

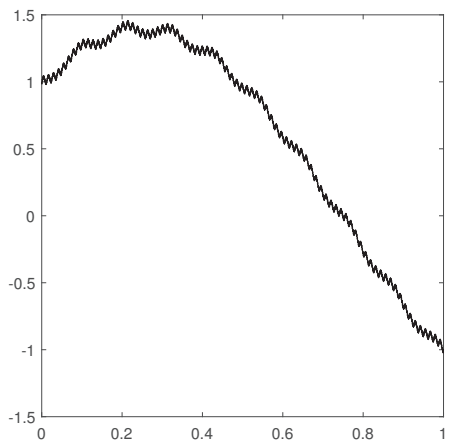

(f): $f^{\alpha}$ with $\alpha_{i}=-0.6, i \in \mathbb{N}_{9}$.

Figure 1. The $\alpha$-fractal approximants of $\sin (\pi x)+\cos (\pi x), x \in[0,1]$.

The $\alpha$-fractal functions $f_{3}^{\alpha}, f_{7}^{\alpha}$ and $f_{26}^{\alpha}$ are generated respectively in Figures 1(b)-(d) with the choice of the scaling factors $\alpha_{i}=0.8, i \in \mathbb{N}_{9}$. Since $f(x)=\sin (\pi x)+\cos (\pi x)$ and Bernstein polynomial $B_{n}(f, x)$ are Lipschitz, it is calculated using [5] that fractal dimension (box dimension) of the $\alpha$-fractal functions $f_{3}^{\alpha}, f_{7}^{\alpha}$ and $f_{26}^{\alpha}$ is 1.8984 . According to Theorem 3.1, the $\alpha$-fractal function $f_{26}^{\alpha}$ shows more similarity with $\sin (\pi x)+\cos (\pi x), x \in[0,1]$ than that of obtained by $f_{3}^{\alpha}$ and $f_{7}^{\alpha}$. By observing Figures $1(\mathrm{~b})-(\mathrm{d})$, one can ask that why are the fractal functions $f_{3}^{\alpha}, f_{7}^{\alpha}$, and $f_{26}^{\alpha}$ not having same sort of irregularity even if they are having same fractal dimension 1.8984? This is due to the following reason: The $\alpha$-fractal functions $f_{3}^{\alpha}$ and $f_{7}^{\alpha}$ exhibit irregularity on all scales whereas the $\alpha$-fractal function $f_{26}^{\alpha}$ exhibits irregularity on small scales. Further, small scales of irregularity of the $\alpha$-fractal function $f_{26}^{\alpha}$ can be observed from Figure 1(e) which is a part of $f_{26}^{\alpha}$ under magnification. From the above discussion and Theorem 3.1, we conclude that even if the scaling factors $\alpha_{i}, i=1,2, \ldots, N-1$, are chosen so that the condition as $\sum_{i=1}^{N-1}\left|\alpha_{i}\right|>1$, then, for large $n \in \mathbb{N}$, the $\alpha$-fractal function $f_{n}^{\alpha}$ may not exhibit irregularity on large scales, but, certainly $f_{n}^{\alpha}$ exhibits irregularity on small scales. If the scaling factors $\alpha_{i}, i \in \mathbb{N}_{N-1}$, are chosen so that the condition as $\sum_{i=1}^{N-1}\left|\alpha_{i}\right|>1$, then for sufficiently large $n, f_{n}^{\alpha}$ provides a good non- 
differentiable approximation to the original function. The $\alpha$-fractal function in Figure 1(h) is generated by taking $\alpha_{i}=-0.6, i \in \mathbb{N}_{9}$.

\section{Acknowledgements}

The author acknowledges the financial support received from Council of Scientific \& Industrial Research (CSIR), India (Project No. 25(0290)/18/EMR-II).

\section{Bibliography}

[1] M. F. Barnsley, Fractal functions and interpolation, Constr. Approx. 2, (1986), 303-329.

[2] A. K. B. Chand and G. P. Kapoor, Generalized cubic spline fractal interpolation functions. SIAM J. Num. Anal. 44(2), (2006), 655-676.

[3] A. K. B. Chand and P. Viswanathan, A constructive approach to cubic Hermite fractal interpolation function and its constrained aspects, BIT Numer. Math. 53 (4), (2013), 841-865.

[4] M. A. Navascués, Fractal polynomial interpolation, Z. Anal. Anwend. 24(2), (2005), 401-418.

[5] MD. Nasim Akhtar, M. Guru Prem Prasad, and M. A. Navascués, Box dimensions of $\alpha$-fractal functions, Fractals 24(3), (2016), 13 pages.

[6] M. A. Navascués, Non-smoothpolynomials, Int .J. Math. Anal. 1, (2007), 159-174.

[7] M. A. Navascués, Fractal approximation, Complex Anal. Oper. Theory 4, (2010), 953-974.

[8] M. A. Navascués, Fractal bases of $L_{p}$ spaces, Fractals 20, (2012), 141-148.

[9] M. A. Navascués and A. K. B. Chand, Fundamental sets of fractal functions, Acta Appl. Math. 100, (2008), 247-261.

[10] M. A. Navascués and M. V. Sebastián, Smooth fractal interpolation, J. Inequal. Appl. (2006), Article ID 78734.

[11] N. Vijender, Bernstein Fractal Trigonometric Approximation, Acta Applicandae Mathematicae 159(1), (2018).

[12] N. Vijender, Bernstein Fractal Rational Approximants with No Condition on Scaling Vectors, Fractals 26(4), (2018).

[13] N. Vijender, Fractal Perturbation of Shaped Functions: Convergence Independent of Scaling, Mediterranean Journal of Mathematics 15(211), (2018).

[14] N. Vijender, Bernstein Fractal Approximation and Fractal Full Müntz Theorems, Electronic Transactions on Numerical Analysis (ETNA) 51, (2019), 1-24 .

[15] S. G. Gal, Shape Preserving Approximation by Real and Complex Polynomials. Birkhäuser, Boston, 2008.

[16] P. Viswanathan, M. A. Navascués, and A. K. B. Chand, Associate fractal functions in $L^{p}$-spaces and in one-sided uniform approximation. J. Math. Anal. Appl. 433 (2016) 862-876.

[17] A. K. B. Chand, M. A. Navascués, P. Viswanathan, and S. K. Katiyar, Fractal trigonometric polynomials for restricted range approximation, Fractals, Vol. 24, No. 2 (2016), 1650022.

[18] P. Viswanathan and A. K. B. Chand, $\alpha$-fractal rational splines for constrained interpolation. Electron. Trans. Numer. Anal. 41, (2014), 420-442.

[19] A.K.B. Chand, S. Jha nd M. A. Navascues Kantorovitch-Bernstein $\alpha$-fractal function in $L_{p}$ spaces Quaestiones Mathematicae, Vol. 43, No.2 (2020).

[20] V. Hutson and J. S. Pym, Applications of Functional Analysis and Operator Theory, Academic Press, New York, 1980. 